\title{
Incidental Idiopathic Chylous Ascites at Cesarean Section
}

\section{Anita Kant ${ }^{1}$ and Amrita Razdan Kaul ${ }^{2 *}$ \\ ${ }^{1}$ Chairman and HOD, Department of Obstetrics and Gynecology, Asian institute of \\ Medical Sciences, Faridabad, Haryana, India \\ ${ }^{2}$ Consultant, Department of Obstetrics and Gynecology, Asian institute of Medical \\ Sciences, Faridabad, Haryana, India}

*Corresponding Author: Amrita Razdan Kaul, Consultant, Department of

Obstetrics and Gynecology, Asian institute of Medical Sciences, Faridabad,

Haryana, India.
Received: October 04, 2021

Published: October 11, 2021

(C) All rights are reserved by Anita Kant and

Amrita Razdan Kaul.

\begin{abstract}
Introduction: Chyloperitoneum or Chylous Ascites refers to the accumulation of lipid rich lymph in peritoneal cavity due to disruption of the lymphatic system secondary to obstruction or trauma. It is rare form of ascites and mostly an incidental finding in pregnancy.

Case-Report: We report a case of chylous ascites in a 31-year old second gravida noted during cesarean section. Milky free fluid was found on opening the peritoneal cavity with no detectable source of trauma or mass. The milky fluid sent for biochemical analysis confirmed presence of triglycerides. Her postoperative course remained uneventful.

Conclusion: Chylous ascites has multiple etiologies including malignancy, liver cirrhosis, intraperitoneal infections and trauma. although only a few cases of chylous ascites in pregnancy have been reported in literature, our case report serves as a reminder for obstetricians to consider possible etiologies of chylous ascites at the time of diagnosis to ensure complete care to mother.
\end{abstract}

Keywords: Idiopathic Chylous Ascites; Pregnancy; Lymph

\section{Introduction}

Chylous ascites is a rare form of ascites with an incidence of 1 in 20000 developing in background of disruption of lymphatic system secondary to multiple etiologies [1] most common being abdominal malignancies and liver cirrhosis accounting for more than two-thirds of the cases in developed countries while intraperitoneal infections and trauma are prevalent particularly in developing countries. Among abdominal malignancies lymphoma account for at least one-third of the cases in one large series of patients identified over 20 years and cirrhosis accounting for two-third of all cases. In contrast infections like tuberculosis, filariasis account for majority of the cases in eastern and developing countries [11]. Congenital, inflammatory, postoperative and traumatic causes are less frequent $[2,3]$.

Chylous ascites is a rare finding during pregnancy with only a few cases reported. Precise mechanism of idiopathic chylous ascites is still unknown [10]. Some cases during pregnancy were found as a complication due to pancreatitis, intestinal volvulus and a few were coincidental finding during cesarean section [12]. Possible causes include exudation of chyle from lymphatic ducts in the wall of the bowel or mesentery due to obstruction of normal lymphatic flow [2]. 


\section{Case Report}

31 year old G2P1L1 patient with height of 5 feet 2 inches and weight of $64 \mathrm{~kg}$ was hospitalized in OBG facility of Asian hospital Faridabad with a POG of 39 weeks 4 days and complaint of labour pains. She has a previous one normal term vaginal delivery 6 years back in same facility. She is been a booked patient with all her prenantal checkups done regularly and uneventful. Her B.P. was within normal limits throughout her antenatal period. Her last ultrasound was done at 37 weeks of gestation with normal findings and without any mention of maternal ascites. After hospitalization with finding of cervical dilatation of $2.5 \mathrm{~cm}$ with partial effacement and admission cardiotocography reactive- augmentation of labour was done with prostaglandins. Her routine blood tests, renal profile (Serum Sodium - 140, Serum Potassium- 4.1), infection profile- all were normal. Her progress of labour was followed. She presented four hours later with fetal distress and vaginal examination was suggestive of same cervical findings hence ARM (artificial rupture of membrane) was done and thick meconium (acute fetal distress) was noted. After informed consent patient was shifted for emergency cesarean section. After opening peritoneal cavity, copious amount of milky white fluid was noted and saved for cytopathology, biochemistry, and microbiology. Vigorous male child weighing $2.8 \mathrm{~kg}$ was delivered. After closing uterine incision complete evaluation of uterus, ovaries and fallopian tubes was done and no pathology found. General surgeon was called in for intraoperative exploration of peritoneal cavity, bowel, liver, and omentum. No suspicious pathology was noted. Thorough peritoneal lavage with normal saline was done and as per surgeon's opinion no drain was kept per-operatively. Postoperatively, the patient was given intravenous antibiotics - the second generation cephalosporin (cefoxitine $1 \mathrm{gm}$ ) and amikacin 750mg until discharge on day 3 of cesarean from hospital. Patient was observed for vitals and bowel movements which remained stable. Analysis of milky fluid was suggestive of non specific inflammation with scattered mononuclear cells on cytopathology, high triglycerides levels of $3866 \mathrm{mg} / \mathrm{dl}$, normal protein and glucose level; negative aerobic cultures on microbiology. High triglyceride level with normal biochemistry with turbid white appearance of fluid confirmed chyloperitoneum or chylous ascites. Patient was advised low fat diet and had uneventful follow up visits after discharge on day-7 and day-45. Acid-fast bacillus culture was also negative.

\section{Discussion}

Chylous Ascites is a finding and not pathology. Exact cause is not clear yet. Various underlying mechanisms have been identified in the literature $[3,10]$.

- Abdominal malignant tumors directly resulting in lymphatic system rupture by invasion or suppression

- Infections blocking the lymph vessels leading to vessel rupture and chylous leak

- Trauma damaging lymph vessels or resulting in chylocyst formation

- Congenital malformations in lymph vessels producing dysplasia, stricture and expansion

- $\quad$ Other diseases such as liver cirrhosis, nephrotic syndrome or mesenteric lymphadenitis resulting in chyle leak from the dilated lymphatic vessels that lack valves [4].

Review of literature has suggested chylous ascites in pregnancy as an extremely rare occurrence with some of the noted associations as follows

- Habek., et al. [5] reported chylous ascites in pregnant patients secondary to pulmonary tuberculosis in childhood with congenital lymphangiectasis.

- Chang., et al. [6] diagnosed chylous ascites in pregnant women with acute abdomen secondary to severe pancreatitis.

- Sun., et al. [7] found chylous ascites incidental to cesarean section in a patient found later with mesenteric tumor in spiral C.T. of abdomen and pelvis. Patient underwent surgical excision of the mass with final histopathology of mesenteric fibromatosis.

- Kondrat'ev [8] reported chylous ascites in pregnant patients with volvulus of small intestine.

- Babic., et al. [9] noted spontaneous ascites in a female with morbid obesity at time of cesarean section.

Evaluating our patient's underlying etiology for spontaneous chylous ascites found incidentally during cesarean section ruled out the above discussed causes. However physiological changes of pregnancy may suggest some of the possible predisposing factors as below 
- $\quad$ Presence of progesterone during pregnancy results in diastolic state of lymphatic ducts with increased flow of lymph through these valve deficient conduits.

- $\quad$ Enlarged gravid uterus in late gestation may suppress the abdominal thoracic duct of mother increasing the pressure of the duct.

Both these changes can result in either spontaneous rupture of the dilated engorged lymph vessels or appearance of giant retroperitoneal lymphatic vessel wall which under the increased pressure during labour result in spontaneous exudation of chyle in the walls of bowel or mesentery.

Significantly elevated levels of triglycerides with rest of normal biochemistry are the most important index confirming the diagnosis of chylous ascites or chyloperitoneum. Idiopathic origin is justified by the fact that no mass or lesion or sign of injury was detectable for any abdominal viscera intraoperatively, no primary disease was diagnosed postoperatively and no pathology could be suspected prenatally for this chylous ascites. Patient recovered without any sequelae further supporting idiopathic nature of this chylous ascites in our case relating to the effect of stasis of progesterone on lymph vessels with increased thoracic duct pressure from the gravid uterus. Hence as delivery corrected the responsible pathology for presence of chyle in peritoneal fluid, patient recovered fully.

\section{Conclusion}

Although chylous ascites is extremely rare in pregnancy, reported cases in literature suggest to be aware of this entity. Diagnosing it timely with attempts to identify the underlying etiology is important for definitive management of the patient.

\section{Bibliography}

1. Yildirim AE., et al. "Idiopathic chylous ascites treated with total parenteral nutrition and octreotide. A case report and review of the literature". European Journal of Gastroenterology and Hepatology 23.10 (2011): 961-963.

2. Yoshimura M. "Lymphoscintigraphy revealed abnormal lymphatic drainage in chyloperitoneum". Clinical Nuclear Medicine 37.1 (2012): e7-e10.

3. Laterre PF., et al. "Chylous ascites: diagnosis, causes and treatment". Acta Gastro-Enterologica Belgica 63.3 (2000): 260-263.
4. Browse NL., et al. "Aetiology and treatment of chylous ascites". British Journal of Surgery 79.11 (1992): 1145-1150.

5. Habek D., et al. "Nontraumatic cyloperitoneum in pregnancy". European Journal of Obstetrics and Gynecology 122 (2005): 243-251.

6. Chuang SC., et al. "Hypertriclyceridemia-associated acute pancreatitis with chylous ascites in pregnancy". Journal of the Formosan Medical Association 105 (2006): 583-587.

7. L Sun., et al. "A rare case of pregnancy complicated by mesenteric mass: what does chylous ascites tell us?" World Journal of Gastroenterology 13.10 (2007): 1632-1635.

8. Kondratev NP. "Small intestine volvulus complicated by acute chylous in pregnant women”. Khirurgiia 2 (1980): 90-91.

9. Babic I., et al. "Spontaneous resolution of chylous ascites following delivery: a case report". Journal of Medical Case Reports 6 (2012): 187.

10. Bili Zhang., et al. "Idiopathic Chylous Ascites in Pregnancy: A case report". Iran Journal of Public Health 47.7 (2018): 10341036.

11. Kida A Thomson. "Incidental Chylous Ascites at the time of Cesarean Section". Case reports in Obstetrics and Gynecology (2015).

12. Liu., et al. "Chylous Ascites in Acute Pancreatitis during pregnancy: Case Report". Chang Gung Medical Journal 24 (2001): 324-328.

\section{Volume 3 Issue 11 November 2021 (C) All rights are reserved by Anita Kant} and Amrita Razdan Kaul. 